\title{
Abnormal Plasma Levels of Serine, Methionine, and Taurine in Transient Acute Polymorphic Psychosis
}

\author{
Durk Fekkes, Lolke Pepplinkhuizen, René Verheij, and Jacques Bruinvels
}

Received September 18, 19,92; revised version received April 22, 1993; accepted September 30, 1993.

\begin{abstract}
The present study explored the usefulness of plasma amino acid concentrations in discriminating a subgroup of patients with transient acute polymorphic psychoses characterized by psychosensory symptoms (APP+ patients). Levels of amino acids in the plasma of APP+ patients were compared with levels in psychiatric patients with other types of psychotic symptomatology and a healthy control group. Both the APP+ patients and patients with bipolar affective disorder had significantly lower plasma concentrations of serine compared with concentrations in the other groups studied. Since the plasma concentrations of taurine and methionine were also different in the APP+ patients, the ratio of taurine to the product of serine and methionine (the TSM ratio) was used in an attempt to increase the sensitivity in discriminating these patients. The TSM ratio in the APP+ patients was significantly higher than those in the other groups studied, except for the patients with bipolar affective disorder. It appears that the determination of serine and the TSM ratio in the plasma of psychotic patients may be a useful diagnostic validator in a group of patients with acute polymorphic psychoses.
\end{abstract}

Key Words. Amino acids, affective disorder, bipolar subtype, schizophrenia.

In 1980, we observed a disturbed serine-glycine metabolism in a subgroup of patients with acute psychosis (Pepplinkhuizen et al., 1980). These transient, but often recurrent, psychotic episodes are characterized by a rich and varying mixture of symptomatology -intense emotional states ranging from overwhelming feelings of ecstasy to terrifying anxiety. Delusions and hallucinations are often minimal in these patients, who often suffer from sensory-perceptual anomalies of the type seen after LSD ingestion (e.g., distorted perceptions of colors, objects, space, body, and time).

In Europe, this form of psychosis has been known as bouffee delirante des degenerées, degeneration psychosis, and cycloid psychosis, in particular, the ecstasyanxiety subform. The $I C D-10$ (World Health Organization, 1992) now classifies these psychoses as acute polymorphic psychotic disorders with or without symptoms of

\footnotetext{
Durk Fekkes, Ph.D., is Chief, Lolke Pepplinkhuizen, Ph.D., is Professor of Biological Psychiatry, and René Verheij, M.D., is Research Fellow, Section on the Pathophysiology of Behavior and Department of Psychiatry, Erasmus University Rotterdam and University Hospital Dijkzigt, Rotterdam, The Netherlands. Jacques Bruinvels, Ph.D., is Professor Emeritus of Pharmacology, Department of Pharmacology, Erasmus University Rotterdam, Rotterdam, The Netherlands. (Reprint requests to Dr. D. Fekkes, Section on the Pathophysiology of Behavior, Room Ee 1371, Medical Faculty, Erasmus University Rotterdam, P.O. Box 1738, 3000 DR Rotterdam, The Netherlands.)
}

0165-1781/94/\$07.00 1994 Elsevier Science Ireland Ltd. 
schizophrenia (F23.0-F23.1). In DSM-III-R (American Psychiatric Association, 1987), these psychotic disorders are categorized as psychotic disorders not elsewhere classified, with particular reference to the group of the schizoaffective (295.7) and schizophreniform disorders (295.40).

Some APP patients report experiencing hallucinogenic drug-like sensory perceptual distortions or "psychedelic experiences." Patients who fulfill the $I C D-10$ criteria for an acute polymorphic psychosis, and who also report distorted sensory perceptions are designated as APP+, and those without perceptual disturbances as APP-. Discriminating this special group is warranted because APP+ patients typically do not respond to standard neuroleptic treatment (Bruinvels and Pepplinkhuizen, 1985), but they do benefit from treatment with the serotonin 2 receptor blocker ritanserin (Pepplinkhuizen, 1988).

In an analogy with the transmethylation hypothesis of schizophrenia, we hypothesized that endogenous hallucinogenic substances might be responsible for the psychotic episodes of APP+ patients (Pepplinkhuizen et al,, 1980). The following observations have linked a disturbed serine (ser) and related folate metabolism to the synthesis of putative hallucinogenic agents in the APP + patients:

1. Oral loading with ser induced the reemergence of characteristic perceptual disturbances and psychedelic symptoms in most recovered APP+ patients (Bruinvels et al., 1988; Pepplinkhuizen et al., 1980); the patients who experienced renewed symptoms in response to ser are termed the "ser positive" group.

2. The plasma concentrations of ser and methionine (met) in APP+ patients were decreased, and the concentration of taurine (tau) was increased (Bruinvels and Pepplinkhuizen, 1984).

3. The activities of the ser-metabolizing enzymes serine hydroxymethyltransferase and cystathionine $\beta$-synthase were increased in eight patients in a pilot study (Fekkes et al., 1991).

On the basis of the above findings, we decided to look at the "TSM ratio," which is defined as the ratio of 100 times the tau concentration in plasma and the product of the plasma concentrations of ser and met. We measured concentrations of the amino acids ser, tau, and met in the plasma of several groups of psychotic patients, including our APP+ patients and a control group, and calculated the TSM ratios. It was hoped that the TSM ratio would prove to be a biochemical marker that could discriminate the APP+ patients with greater sensitivity and specificity than the plasma ser concentration alone. If this proved to be the case, the TSM ratio might eventually replace the more invasive and burdensome ser loading test.

\section{Methods}

Subjects. We evaluated 22 healthy control subjects (group g; see Table 1) who were randomly selected from among our hospital personnel ( 12 men and 10 women; aged 21 to 48 years) and 94 patients ( 47 men and 47 women; aged 19 to 53 years) who were admitted for an acute psychotic episode. With the exception of four bipolar patients, the patients had not yet been treated with antipsychotics during the month before the present admission. The final diagnostic subdivision of these 94 patients was as follows: (a) 18 acute psychotic patients 
Table 1. Characteristics of psychotic patients and healthy control subjects

\begin{tabular}{lccc}
\hline Diagnostic category & $\begin{array}{c}\text { Sex } \\
\text { (female/male) }\end{array}$ & $\begin{array}{c}\text { Challenge } \\
\text { test }^{\text {1 }}\end{array}$ & $\begin{array}{c}\text { Psychopathological } \\
\text { reaction }\end{array}$ \\
\hline $\begin{array}{l}\text { APP', perceptual disturbances } \\
\text { present (APP+; a+b) }\end{array}$ & $16 / 12$ & 18 & 18 \\
APP, no perceptual disturbances & & & \\
$\quad$ present (APP-; c) & $12 / 8$ & 10 & 0 \\
Atypical psychosis (d) & $9 / 3$ & 3 & 0 \\
Bipolar affective disorder (e) & $8 / 8$ & 9 & 0 \\
Schizophrenia (f) & $6 / 12$ & 6 & 0 \\
Healthy control subjects (g) & $10 / 12$ & 15 & 0 \\
\hline
\end{tabular}

1. The challenge test was performed by giving the subjects an oral dose of serine $(2 \mathrm{mmol} / \mathrm{kg}$ body weight).

2. APP = acute polymorphic psychosis. See Methods for explanation of groups a-g.

suffering from APP+, all "ser positive" (Table 1); (b) 10 APP+ patients who refused to undergo the ser-loading challenge; (c) 20 patients suffering from transient acute polymorphic psychosis without psychosensory symptoms (APP-); (d) 12 patients suffering from atypical psychoses according to $D S M-I I I-R$ criteria (298.90); (e) 16 patients with bipolar affective disorder, of whom four were taking lithium medication; and (f) 18 schizophrenic patients. For an over view, see Table 1, which also shows the results of the ser challenge test performed in a number of patients and healthy subjects.

Biochemical Determinations. Blood was drawn by venipuncture in heparinized tubes between 8 and 9 a.m. before breakfast. Blood sampling in the patients was done when psychotic symptoms were fully present and before the beginning of treatment with antipsychotic medication. The plasma was immediately separated by centrifugation for 5 minutes at $1560 \mathrm{~g}_{\max }$ and stored at $-30^{\circ} \mathrm{C}$. Within 3 months, $500 \mu \mathrm{l}$ of plasma were deproteinized with $100 \mu \mathrm{l}$ of $25 \%(\mathrm{w} / \mathrm{v})$ sulfosalicylic acid containing a known amount of the internal standard norleucine. The amino acids in these samples were measured by ionexchange chromatography on an LKB 4400 Amino Acid Analyzer with fluorescence detection (Bruinvels and Pepplinkhuizen, 1984). The ratio of the tau concentration and the product of the concentrations of ser and met was calculated and multiplied by 100 (TSM ratio).

Statistics. Variables were tested for homogeneity of variances by means of Cochran's $C$ test. Multivariate analysis of variance (MANOVA) was used to test for differences in amino acid concentrations and TSM ratios across groups, followed by univariate analysis of variance (ANOVA) and Student-Newman-Keuls procedure for individual variables. In addition, Pearson's correlation coefficients were calculated to investigate the relations between the amino acid concentrations and the TSM ratio. The significance level $\alpha$ was set at 0.05 (two-tailed).

\section{Results}

All amino acid data were normally or log-normally distributed. Data for one bipolar patient were discarded, because both ser and met concentrations for this patient were extremely high. Table 2 lists the plasma levels of ser, tau, and met of several groups of patients and the control group. Group differences for the multivariate dimension were statistically significant (MANOVA: $F=4.05 ; d f=20,418 ; p<0.001$ ). Univariate tests showed that group differences existed for $\operatorname{ser}(F=6.68 ; d f=5,109$; $p<0.0001)$, met $(F=4.11 ; d f=5,109 ; p=0.0019)$, and the TSM ratio $(F=12.38$; 


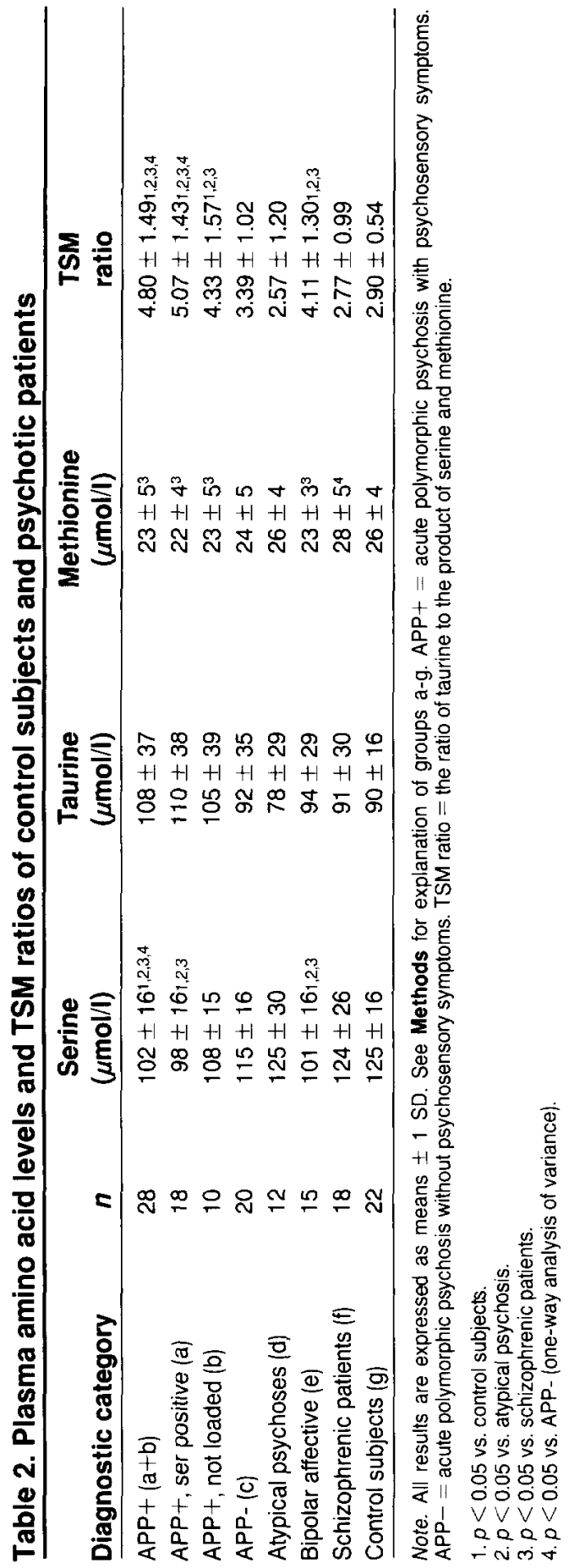


$d f=5,109 ; p<0.0001)$, but not for tau $(F=1.88 ; d f=5,109 ; p=0.103)$. With the Student-Newman-Keuls procedure for individual variables, it was found that the APP + patients (groups $a+b$ ) showed significantly lower plasma ser levels compared with control subjects (group g; see Fig. 1), APP- patients (group c), schizophrenic patients (group f), and patients with atypical psychoses (group d). The plasma ser concentration (mean $\pm 1 \mathrm{SD}$ ) of the 18 ser-positive patients (group a; $98 \pm 16 \mu M$ ) was slightly lower than the ser level in the plasma of the APP+ patients who refused to participate in the loading experiment (group $b ; 108 \pm 15 \mu M$ ). No differences were found between APP+ patients and patients with bipolar affective disorder (group e). The plasma level of $\operatorname{ser}(101 \pm 16 \mu M)$ in the bipolar patients was significantly lower than in the control subjects, the schizophrenic patients, and the atypical psychotic patients.

The plasma levels of tau showed no significant differences between groups, although the tau concentration in the APP + patients was slightly higher than in the other groups studied. The plasma levels of met in both APP+ and APP- patients did not differ from the values in the control group or in the bipolar and atypical

Fig. 1. Scatter plots of serine concentrations (in $\mu \mathrm{mol} / \mathrm{l}$ ) and TSM ratios in blood plasma of $28 \mathrm{APP}+$ patients and 22 healthy control subjects
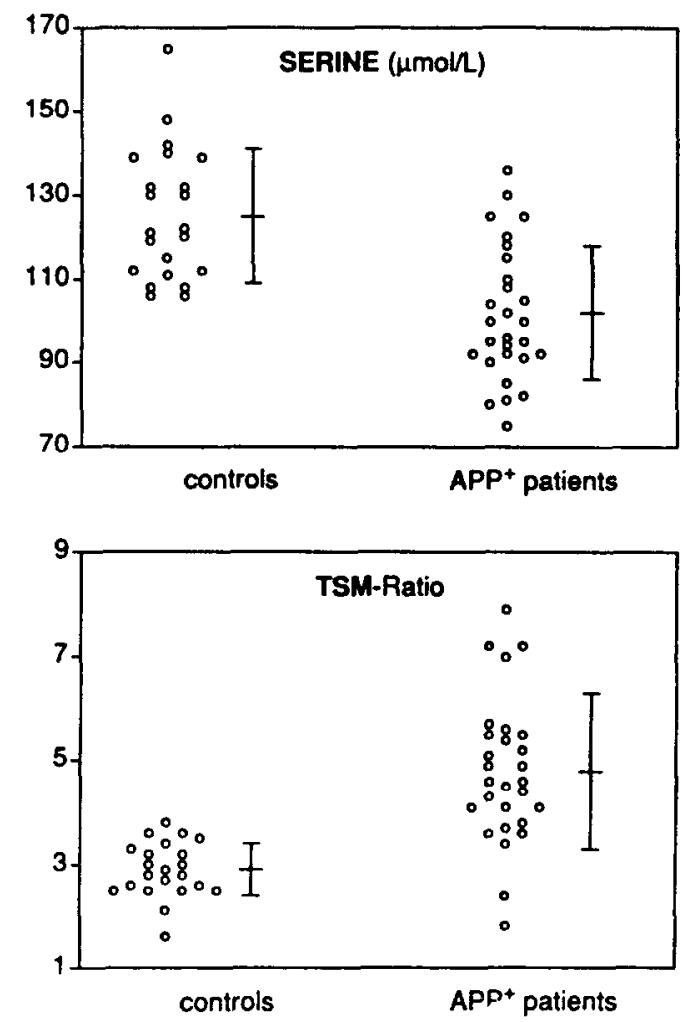

The error bars indicate the mean and SD. TSM ratio = the ratio of taurine to the product of serine and methionine. $\mathrm{APP}+=$ acute polymorphic psychosis with psychosensory symptoms. 
psychosis patients. However, the met concentration in the plasma of the schizophrenic patients was significantly higher than in the plasma of APP+ patients, APP - patients, and bipolar affective patients.

Table 2 also displays the TSM ratios. The ratio in the ser-positive patient group was significantly higher than the ratio in the control group (Fig. 1) or in the other groups of psychiatric patients except for the bipolar patients. The same was true for all APP+ patients. The TSM ratio in the bipolar patient group was significantly higher than in the atypical psychotic patients, the schizophrenic patients, or the control subjects. No difference in TSM ratio was found between the control subjects and the schizophrenic, atypical psychotic, or APP- patients.

The plasma tau concentration was positively correlated with the TSM ratio in every single patient group studied $(r>0.54, p<0.02)$, but not in the control subjects $(r=0.25, p=0.225)$. Another interesting finding was that the plasma tau level correlated positively with the plasma met concentration only in the APP patients, irrespective of the presence of psychedelic symptoms $(r>0.53, p \leqslant 0.003)$.

\section{Discussion}

The plasma levels of ser and met in the group of healthy control subjects in this study are in agreement with those reported in other studies (Bjerkenstedt el al., 1985; Waziri and Mott, 1986; Rao et al., 1990). However, the plasma concentration of tau was higher than in most other studies. This is due to the short centrifugation time (5 minutes) and the relatively low centrifugal speed $(1560 \mathrm{~g})$ that we used for the plasma preparation. This yields plasma containing high amounts of blood platelets, which are known to be rich in the amino acid tau.

As in our previous studies (Bruinvels and Pepplinkhuizen, 1984, 1985), we found significantly lower plasma ser concentrations in APP + patients than in control subjects. The plasma tau concentration in these patients was - as in an earlier study (Bruinvels and Pepplinkhuizen, 1984) - slightly elevated, although not to a statistically significant degree. The bipolar affective patients also had decreased plasma ser concentrations. This is in contradiction to an earlier study (Bruinvels and Pepplinkhuizen, 1984), although that study included only three bipolar patients. Psychopharmacologic treatment was not the cause of these low plasma ser levels, since exclusion of the data of the medicated patients resulted in comparable (102 士 $17 \mu M$ ) plasma ser concentrations.

The TSM ratio discriminated the APP+ patients with greater sensitivity $(F=12.38)$ than did the plasma ser concentration alone $(F=6.68)$. However, the specificity was not better, because the TSM ratio in the bipolar affective patients was only slightly lower $(4.11 \pm 1.30)$ than in the APP+ patients $(4.80 \pm 1.49)$.

Plasma ser levels did not differ among control subjects, patients suffering from atypical psychoses, and schizophrenic patients. This finding is in agreement with the results reported by Perry and Hansen (1985), Bjerkenstedt et al. (1985), and Rao et al. (1990) for schizophrenic patients. Although the plasma ser levels in the normal control subjects $(125 \mu M)$ were comparable to those published by Waziri and Mott $(1986,117 \mu M)$, the very high ser concentration of $205 \mu \mathrm{M}$ in the plasma of psychotic patients reported by these investigators was not found in our patients. The plasma 
ser concentration of $133 \mu M$ in the schizophrenic patients studied by Macciardi et al. (1990) was comparable to the value that we found $(123 \mu M)$. However, the plasma ser level in the control group studied by Macciardi et al. was lower $(109 \mu M)$ than that in our control group $(125 \mu M)$. The possibility of a drug effect on plasma ser concentrations can be ruled out, as omission of the data of medicated patients did not result in higher ser levels. This is in accordance with the results of Rao et al. (1990), who reported that medication had no effect on serum ser levels in schizophrenic patients.

Concerning the plasma met concentrations, we found no differences between schizophrenic patients and control subjects. This is in line with the results reported by Rao et al. (1990) but in contradiction to those reported by Bjerkenstedt et al. (1985) and Macciardi et al. (1990), who both found increased levels of met in the plasma of schizophrenic patients.

The explanation for the discrepancies between the plasma ser concentrations in schizophrenic patients reported by different investigators is unclear. Possibly, a combination of several slightly differing factors among the studies (e.g., techniques of blood withdrawal, processing, storage and amino acid analysis, dietary intake, gender, diagnosis, and medication) accounts for these discrepancies. The major finding of this study is that a decreased plasma ser concentration and an increased TSM ratio both are at least state markers for the subgroup of episodic psychotic (APP+) patients (Fig. 1). However, these markers cannot differentiate between APP+ patients and bipolar affective patients. An investigation of the TSM ratio as a trait marker is in progress.

In conclusion, a suggestive diagnosis can be based on the presence of an episodic acute psychosis with sensory perceptual distortions and psychedelic symptoms, a kaleidoscopic polymorphic clinical picture, a low plasma serine concentration, and a high TSM ratio. However, confirmation of this diagnosis requires the development of characteristic symptoms after oral challenge with serine.

Acknowledgments. We thank Mrs. A. van Straten for technical assistance and Mrs. J. Zaanen for typing the manuscript. This study was supported by a grant from "Praeventiefonds," No. 28-753.

\section{References}

American Psychiatric Association. DSM-III-R: Diagnostic and Statistical Manual of Mental Disorders. 3rd ed., revised. Washington, DC: American Psychiatric Press, 1987.

Bjerkenstedt, L.; Edman, G.; Hagenfeldt, L.; Sedvall, G.; and Wiesel, F.A. Plasma amino acids in relation to cerebrospinal fluid monoamine metabolites in schizophrenic patients and healthy controls. British Journal of Psychiatry, 147:276-282, 1985.

Bruinvels, J., and Pepplinkhuizen, L. Impaired glycine-serine conversion and increased plasma taurine levels in episodic psychotic patients with psychedelic symptoms. Journal of Psychiatric Research, 18:307-318, 1984.

Bruinvels, J., and Pepplinkhuizen, L. Disturbances in serine-glycine metabolism in relation to acute psychoses with psychedelic symptoms. In: Beckmann, H., and Riederer, P., eds. Pathochemical Markers in Major Psychoses. Berlin: Springer, 1985. pp. 59-73.

Bruinvels, J.; Pepplinkhuizen, L.; and Fekkes, D. Derangement of one-carbon metabolism in episodic schizoaffective psychoses. Pharmacopsychiatry, 21:28-32, 1988. 
Fekkes, D.; Pepplinkhuizen, L.; and Bruinvels, J. Changes in serine metabolism by a serum factor present in a group of episodic psychotic patients. Biological Psychiatry, 30:966-972, 1991.

Macciardi, F.; Lucca, A.; Catalano, M.; Marino, C.; and Smeraldi, E. Amino acid patterns in schizophrenia: Some new findings. Psychiatry Research, 32:63-68, 1990.

Pepplinkhuizen, L. Ritanserin in the treatment of therapy resistent psychoses: 13 pilot case reports. Janssen Research Report, R 55 667-NL, 1988.

Pepplinkhuizen, L.; Bruinvels, J.; Blom, W.; and Moleman, P. Schizophrenia-like psychosis caused by a metabolic disorder. Lancet, I:454-456, 1980.

Perry, T.L., and Ilansen, S. Interconversion of serine and glycine is normal in psychotic patients. Psychiatry Research, 15:109-113, 1985.

Rao, M.L.; Gross, G.; Strebel, B.; Bräunig, P.; Huber, G.; and Klosterkötter, J. Serum amino acids, central monoamines, and hormones in drug-naive, drug-free, and neuroleptictreated schizophrenic patients and healthy subjects. Psychiatry Research, 34:243-257, 1990.

Waziri, R., and Mott, J. Drug effects on serine metabolism in psychiatric patients. Psychiatry Research, 18:119-126, 1986.

World Health Organization. ICD-10 Classification of Mental and Behavioural Disorders. Geneva: WHO, 1992. 